\title{
IL KEYNES DI GIORGIO LA MALFA: UNA NOTA
}

\author{
di Renata Targetti Lenti
}

Nell'aprile 2019 è stato pubblicato, a cura di Giorgio La Malfa, un "Meridiano" Mondadori che raccoglie scritti di John Maynard Keynes (1883-1946) ${ }^{1}$. Si tratta di una pubblicazione davvero eccezionale, dal momento che sono pochi i "Meridiani" che non siano dedicati a grandi autori della letteratura e della filosofia. Giorgio La Malfa ha compiuto gli studi di economia a Cambridge come allievo di alcuni eredi di Keynes (Khan, i due Robinson, Meade e anche Piero Sraffa) e ha completato la propria formazione di economista presso il Massachusetts Institute of Technology di Boston collaborando con Franco Modigliani: è stato poi professore ordinario di Politica economica nell'Università di Catania. In precedenza, una decina di anni fa, ha curato un altro scritto di Keynes, Sono un liberale? e altri scritti2 ${ }^{2}$, ha scritto inoltre la prefazione alla traduzione di Le mie prime convinzioni ${ }^{3}$, e ha pubblicato una biografia dell'economista inglese ${ }^{4}$. Giorgio La Malfa ha lavorato per diversi anni alla pubblicazione di questo nuovo testo, traducendo personalmente la Teoria generale. Il volume contiene inoltre altri scritti, sei precedenti e venti successivi all'opera principale, utili tutti

Università di Pavia.

1 J.M. KeYnes, Teoria generale dell'occupazione, dell'interesse e della moneta e altri scritti, traduzione e introduzione di Giorgio La Malfa, Milano, I Meridiani Mondadori, 2019, pp. 1328.

2 J.M. Keynes, Sono un liberale? e altri scritti, a cura di Giorgio La Malfa, Milano, Adelphi, 2010.

3 J.M. KEYNes, Le mie prime convinzioni, prefazione di Giorgio La Malfa, Milano, Adelphi, 2012.

4 G. La Malfa, Keynes. Keynes visto da Giorgio La Malfa, Roma, Luiss University Press, 2006. 
a facilitarne la comprensione, nonchè un'ampia cronologia della vita di Keynes. La maggior parte di questi scritti non era mai stata tradotta in italiano. Si segnala, in particolare la Lettera aperta al presidente Roosevelt 5 scritta a pochi mesi dall'elezione di questi, uno scritto di singolare attualità come L'Arte e lo Stato 6 e il saggio su Come pagare il costo della guerra ${ }^{7}$ del 1940, nel quale Keynes anticipa l'idea delle politiche dei redditi. Ancora di La Malfa, con Giovanni Farese, sono le note di commento. Si tratta - sottolineo - di un unicum storico, la sola edizione della Teoria generale criticamente annotata.

Particolarmente stimolante e ricco di osservazioni è il saggio introduttivo Saggezza nuova per una nuova era ${ }^{8}$, nel quale La Malfa sottolinea come Keynes non sia stato solo un economista, ma anche colui che ha ricondotto l'economia nell'ambito delle scienze sociali e morali. Il vero economista infatti, secondo Keynes, è chiamato, prima ancora che a individuare leggi matematiche, a collaborare alla costruzione di un umanesimo adeguato al mondo moderno. Un capitalismo temperato da interventi pubblici, nel contesto di un ordine politico liberaldemocratico, è per lui la migliore soluzione possibile dei problemi della convivenza umana. Un Keynes, quindi, non solo studioso geniale, ma ricco di umanità che ha rovesciato, alle soglie della seconda guerra mondiale, il predominio dell' indirizzo dominante nei cinquant'anni precedenti: cioè una concezione dell'economia che aveva l'obiettivo di assimilarla alle "vere scienze", cioè le scienze della natura. Keynes era infatti convinto, come scrive nel 1938 in una lettera a Harrod, che "l'economia è essenzialmente una scienza morale, non una scienza naturale. Impiega cioè l'introspezione e i giudizi di valore" 9 .

Keynes è stato una figura di intellettuale "assai variegata, il cui orientamento nei confronti degli studi economici si era nutrito di una solida

5 J.M. KEYNES, Teoria generale dell'occupazione, dell'interesse e della moneta e altri scritti, cit., pp. 554-569.

6 J.M. KEYNES, Teoria generale dell'occupazione, dell'interesse e della moneta e altri scritti cit., pp. 581-589.

7 J.M. KEYNES, Teoria generale dell'occupazione, dell'interesse e della moneta e altri scritti, cit., pp.756-840.

8 J.M. KEYNES, Teoria generale dell'occupazione, dell'interesse e della moneta e altri scritti, cit., pp. IX- CXII.

9 J.M. KEYNES, Teoria generale dell'occupazione, dell'interesse e della moneta e altri scritti, cit., p. CLXXXIII. 
preparazione matematica e di una riflessione approfondita su alcune questioni filosofiche, affiancate da un vivo interesse per la psicologia e la psicoanalisi, nonché da una spiccata sensibilità per l'arte e la letteratura" 10 . Quando, all'inizio del 1908, a Keynes venne negata una fellowship al King's College, il giudizio espresso su di lui dal matematico Whitehead, che non era certo uno studioso di secondo piano, fu il seguente: "Mi permetto di dubitare che il signor Keynes abbia sufficientemente colto la differenza fra lo stile proprio della letteratura e quello adatto all'investigazione logica e scientifica; [...] il risultato è tale da creare delle perplessità in un rozzo logico che ha soltanto l'ansia di stabilire quale sia di fatto il vero sviluppo e la base dell' argomentazione" 11. Era invece proprio questa capacità di non restare confinato in un campo ristretto quello che faceva di Keynes uno di quei rarest of birds che sono i grandi economisti12. "L'ostilità di Keynes verso la matematica non era ostilità verso le cifre... nella visione keynesiana la matematica può essere utilizzata quando le condizioni in cui si opera lo permettono, tenendo conto della complessità del reale"13. Robbins, liberale ortodosso e non certo ammiratore delle dottrine keynesiane, lo ritraeva così: “...mi trovo a pensare che Keynes sia probabilmente uno degli uomini più notevoli che siano mai esistiti: la logica pronta, il balzo alato dell'intuizione, la vivida fantasia, l'ampia visione delle cose, soprattutto il senso incomparabile della parola giusta, tutto concorre a farne qualcosa di ben superiore al normale livello umano"14. Per Bertrand Russell "Keynes aveva la mente più acuta e più limpida che mai abbia incontrato. Quando discutevo con lui ero costretto a impegnarmi al massimo e di rado ne uscivo senza l'impressione di avere fatto la figura dello sciocco"15.

10 J.M. KEYNES, Teoria generale dell'occupazione, dell'interesse e della moneta e altri scritti, cit., p. XV.

11 J.M. KEYNES, Teoria generale dell'occupazione, dell'interesse e della moneta e altri scritti, cit., p. CV. Il giudizio è di A.M. CARABELl, On Keynes' Method, London, Macmillan, 1988, p. 10.

12 J.M. KEYNES, Teoria generale dell'occupazione, dell'interesse e della moneta e altri scritti, cit., p. CVI.

13 J.M. KEYNES, Teoria generale dell'occupazione, dell'interesse e della moneta e altri scritti, cit., p. XCIX.

14 J.M. KEYNES, Teoria generale dell'occupazione, dell'interesse e della moneta e altri scritti, cit., p. CXC.

15 J.M. KEYNES, Teoria generale dell'occupazione, dell'interesse e della moneta e altri scritti, cit., p. CXCIV. 
La nuova edizione dei "Meridiani", con il suo ricco corredo di note, contiene una fitta rete di citazioni evidenziando non solo riferimenti storici e dottrinali, ma anche i profondi legami intellettuali fra Keynes e pensatori del calibro di Freud e Wittgenstein, fisici e matematici come Einstein, scrittori come Ibsen, Eliot e Virginia Woolf, politici come Roosevelt, ma anche economisti non ortodossi come Sraffa. Alcune di queste citazioni rivelano l'avversione di Keynes per i teorici dell'individualismo metodologico. Secondo Keynes, infatti, l'analisi tradizionale, dei marginalisti "è sbagliata perché non è riuscita a individuare correttamente le variabili indipendenti del sistema. Risparmio e investimento sono infatti determinati dal sistema, non ne sono le determinanti" "16. Traendo ispirazione dall'Anitra selvatica di Ibsen, Keynes, nel capitolo 14 della Teoria generale, paragona gli economisti marginalisti all'anatra della leggenda nordica che, una volta ferita, si tuffa verso il fondo quanto più può e si afferra "con il becco alle alghe e ai rifiuti che stanno laggiù, e servirebbe un cane straordinariamente abile per tuffarsi e ripescarla dal fondo" 17 .

Freud è un'altra fonte di ispirazione per molti dei concetti innovativi della Teoria generale, anche se non è sempre citato. Sono infatti gli animal spirits che spiegano il comportamento degli imprenditori. La propensione al consumo dipende da fattori oggettivi (reddito, ricchezza, rapporto di scambio tra beni presenti e beni futuri), ma anche da fattori soggettivi, di natura psicologica, cioè da quegli incentivi individuali e sociali che determinano l'ammontare della spesa. In particolare l'astensione dal consumo dipende dal desiderio di lasciare un patrimonio alle generazioni future, ma anche "per soddisfare la propria spilorceria cioè un'avversione, tanto irragionevole quanto tenace, nei confronti di qualsiasi atto di spesa in sé e per sé..... A questi otto motivi si possono dare i nomi di Precauzione... Orgoglio e Avarizia"18. Particolarmente interessante è inoltre il riferimento alla cupidigia umana nei confronti dell'oro tipica del gold standard negli anni Trenta, che si traduce in una accumulazione improduttiva di ricchezza. Il riferimento,

16 J.M. KEYNES, Teoria generale dell'occupazione, dell'interesse e della moneta e altri scritti, cit., p. 211.

${ }^{17}$ H. IBSEN, L'anitra selvatica, Torino, Einaudi, 1963, p. 47 e p. 49.

18 J.M. KEYNES, Teoria generale dell'occupazione, dell'interesse e della moneta e altri scritti, cit., p. 128. 
in questo caso, è al re Mida "il quale non può nutrirsi perché tutto quello che tocca si trasforma in oro"19.

Infine anche da Einstein, che Keynes conosceva personalmente, egli trae un paragone molto efficace. Gli economisti "classici" vengono definiti "geometri euclidei in un mondo non euclideo, i quali, scoprendo che nell'esperienza linee rette apparentemente parallele spesso si incontrano, rimproverano alle linee di non mantenersi diritte...il vero rimedio ...è gettare a mare l'assioma delle parallele ed elaborare una geometria non euclidea. Qualcosa di simile è oggi necessario in economia" 20 . Da queste brevi citazioni appare chiaro quanto Keynes fosse consapevole della portata rivoluzionaria del proprio lavoro, collocabile nell'ambito delle altre rivoluzioni che si stavano sviluppando a quel tempo, in particolare nella morale, nella filosofia, nella psicanalisi e nella fisica. Non "è ingiustificato ipotizzare che Keynes ritenesse il proprio contributo alla 'generalizzazione' dei risultati economici analogo a quello che Einstein aveva messo in campo per la fisica" 21.

La critica radicale al laissez faire, alla teoria economica classica basata sulla convinzione che esistano leggi di mercato capaci di imporre un'autoregolamentazione degli scambi, dei valori e dei prezzi, alle quali è assurdo opporsi, discende dalla convinzione che l'uomo sia chiamato a regolare il mondo dove vive e soprattutto ne sia responsabile nei confronti della società. Nonostante una vita caratterizzata da un accentuato anticonformismo, tipico delle élites britanniche della prima metà del Novecento, Keynes aveva profondo il senso morale e il rispetto per la tradizione religiosa. In una conversazione con Thomas Eliot ebbe a dire: "Comincio a rendermi conto che la nostra - la mia e la tua - generazione deve molto alla religione dei nostri padri. I giovani che crescono senza di essa non riusciranno a vivere appieno la loro vita"22.

Keynes viveva in parte a Cambridge, ma in parte trascorreva il

19 J.M. KEYNES, Teoria generale dell'occupazione, dell'interesse e della moneta e altri scritti, cit., p. CLV.

20 J.M. KEYNES, Teoria generale dell'occupazione, dell'interesse e della moneta e altri scritti, cit., p. 26.

21 J.M. KEYNES, Teoria generale dell'occupazione, dell'interesse e della moneta e altri scritti, cit., p. LXVI.

22 J.M. KEYNES, Teoria generale dell'occupazione, dell'interesse e della moneta e altri scritti, cit., p. CLXXV. 
tempo a Londra, con Virginia Woolf e gli altri amici del Circolo di Bloomsbury, passando le serate assistendo ai balletti, scrivendo per i giornali, giocando in Borsa. Fu anche un eccellente diplomatico. Al termine della prima guerra mondiale partecipò alla Conferenza di pace di Parigi del 1919 come principale funzionario del Tesoro "sostituto del Cancelliere dello Scacchiere nel Supremo Consiglio economico con pieni poteri di decisione"23. Così scriveva nel dicembre 1919: "Se miriamo a impoverire l'Europa centrale, la vendetta, oso predire, non si farà attendere. Niente allora potrà ritardare a lungo quella finale guerra civile tra le forze della reazione e le convulsioni disperate della rivoluzione, rispetto alla quale gli orrori della passata guerra tedesca svaniranno nel nulla e che distruggerà, chiunque sia il vincitore, la civiltà e il progresso della nostra generazione"24. In occasione della Conferenza Keynes conobbe "Carl Melchior un banchiere ebreo tedesco al quale dedicherà un bellissimo ritratto pubblicato soltanto postumo"25. Nel 1923 andò nuovamente a Berlino ospite di Carl Melchior. In un incontro con il Ministro degli Esteri tedesco egli "suggerisce la risposta che la Germania dovrebbe dare alle varie richieste degli Alleati in tema di riparazioni. Dell'argomento ha discusso prima di partire con il governo inglese, convincendolo ad accogliere favorevolmente l'eventuale nota tedesca. A questa iniziale apertura non seguirà però alcuna iniziativa. In Germania si aggrava la crisi del marco, mentre l'inflazione è alle stelle"26. Purtroppo Keynes rimase inascoltato da parte dei vincitori, che vollero vendicarsi della Germania sconfitta esigendo debiti di guerra che non sarebbero mai stati pagati, ma creando in tal modo una situazione post-bellica fortemente squilibrata e foriera dei drammi che avrebbero portato al secondo conflitto mondiale. Keynes fu anche pre-

23 J.M. KEYNES, Teoria generale dell'occupazione, dell'interesse e della moneta e altri scritti, cit., p. CXLIII.

24 J.M. Keynes, The Economic Consequences of the Peace, London, Macmillan, 1919 (trad. it. Le conseguenze economiche della pace, traduzione di F. Salvatorelli, Milano, Adelphi, 2007, p. 212).

25 J.M. KEYNES, Teoria generale dell'occupazione, dell'interesse e della moneta e altri scritti, cit., p.CXLIII. Il ritratto è contenuto in J.M. KEYNES, Le mie prime convinzioni (traduzione di P. Dacrema e B. Bruno, a cura di D. Garnett, con un saggio introduttivo di G. La Malfa) Milano, Adelphi, 2012.

26 J.M. KEYNES, Teoria generale dell'occupazione, dell'interesse e della moneta e altri scritti, cit., p. CLIII. 
sente, nel 1944, alla conferenza di Bretton Woods che definì l'assetto economico del dopoguerra, dove però non riuscì a far passare la propria idea di Clearing Union, con una Banca Centrale che avrebbe gestito un sistema fondato su una nuova moneta, il bancor, slegata dalle altre divise e dall'oro. Gli Stati Uniti si accordarono tuttavia con gli inglesi per un sistema di cambi fissi basato sulla parità delle monete rispetto al dollaro, portando a garanzia le proprie riserve auree, che detenevano in enorme quantità 27 .

Nella lunga e approfondita introduzione al volume Giorgio La Malfa riflette su quanto l'aggettivo "generale" nel titolo dell'opera maggiore dello studioso inglese debba essere collegato alla denominazione della forma definitiva della teoria della relatività, definita appunto come "generale". La generalità della nuova teoria consiste infatti, secondo Keynes, nel ridurre la teoria economica classica a un semplice caso particolare, caratterizzato dalla situazione di piena occupazione. E proprio l'occupazione è la questione che più di altre interessa lo studioso, in quanto collegata alla parte debole della società, che paga il prezzo delle crisi, e di fronte alla quale non si può semplicemente sostenere, come fa la teoria del laissez faire, che prima o poi le crisi saranno superate e che il mercato aggiusterà tutto. Per Keynes la piena occupazione è la precondizione per il mantenimento della democrazia e della libertà nei paesi capitalisti maturi. Una disoccupazione elevata in presenza di forti indennizzi ai disoccupati, come accade oggi nei paesi europei, genera pressioni che divengono intollerabili per il bilancio pubblico e i redditi degli occupati che devono mantenere i disoccupati. Nell'ultimo capitolo della Teoria generale Keynes scrive infatti: "La critica che abbiamo mosso alla teoria economica classica nella sua versione correttamente accettata è consistita non tanto nell'individuarvi errori logici, quanto nel porre in rilievo che essa si basa tacitamente su presupposti che non sono mai o quasi mai soddisfatti, e che di conseguenza non è in grado di risolvere i problemi economici del mondo reale" 28 .

La Teoria generale è un libro "difficile", non perché vi siano impiegati particolari termini tecnici o sia stata scritta in un linguaggio

27 J.M. KEYNES, Teoria generale dell'occupazione, dell'interesse e della moneta e altri scritti, cit., p. CXCI.

28 J.M. KEYNES, Teoria generale dell'occupazione, dell'interesse e della moneta e altri scritti, cit., p. 433. 
complicato. Al contrario, la prosa di Keynes è molto scorrevole. È invece difficile per il contenuto, eterodosso rispetto alla teoria dominante e assolutamente innovativo, tanto da meritare la definizione di "Rivoluzione keynesiana". L'idea che il livello della produzione non fosse determinato dall'offerta, ma dalla domanda di investimenti e di consumi, suonò infatti nel 1936, anno della pubblicazione dell'opera, come un attacco alla "cittadella dell'ortodossia", ai princìi della dottrina classica. Lo stesso Keynes scriveva nel 1935 in una lettera a George Bernard Shaw: "Sto scrivendo un libro sulla teoria economica che, ritengo, rivoluzionerà in larga misura - non subito, immagino, ma nel giro dei prossimi dieci anni - il modo in cui il mondo riflette sui problemi economici...per parte mia, non soltanto spero in quello che dico - ne ho l'assoluta certezza interiore"29.

Proprio questo suo carattere "rivoluzionario" spiega le riserve rivolte alla Teoria generale da parte di molti economisti importanti in Inghilterra, ma soprattutto in Italia, contrariamente a quanto era avvenuto per le opere precedenti, e negli Stati Uniti. Una prima obiezione ben argomentata alla tesi contenuta nel Treatise on Money, secondo cui uno squilibrio tra risparmio e investimento avrebbe comportato solo variazioni dei prezzi relativi e non anche nella produzione dei beni di consumo e di investimento, gli venne da Ralph Hawtrey ${ }^{30}$. Keynes rispose ai dubbi di Hawtrey con un lungo memorandum, il 28 novembre 1930. La riflessione di Keynes prende le mosse proprio dal "superamento della divisione tra le questioni legate alla produzione e quelle di carattere monetario, trattando insieme la teoria monetaria e la teoria dell'offerta di breve periodo - cioè della produzione" 31 . In un primo tempo altri giovani economisti che poi diventeranno keynesiani, come Richard Kahn, Joan e Austin Robinson, James Meade e Piero Sraffa, finirono con il condividere le riserve di Hawtrey ${ }^{32}$. Queste obiezioni convinsero Keynes a "concludere che non si potesse presupporre che

29 J.M. KEYNES, Teoria generale dell'occupazione, dell'interesse e della moneta e altri scritti, cit., p. XII

30 J.M. KEYNES, Teoria generale dell'occupazione, dell'interesse e della moneta e altri scritti, cit., p. XXX.

31 J.M. KEYNES, Teoria generale dell'occupazione, dell'interesse e della moneta e altri scritti, cit., pp. XXX-XXXI.

32 J.M. KEYNES, Teoria generale dell'occupazione, dell'interesse e della moneta e altri scritti, cit., p. XXXI. 
il livello dell'attività produttiva fosse dato indipendentemente dall'andamento dei prezzi e dunque che non fosse possibile tenere separata l'analisi dei due fattori' 33 . Nel 1931 scrisse a Nicholas Kaldor che era pronto a correggere le basi teoriche delle proprie opinioni abbandonando l'economia dello scambio reale in cui la moneta fosse esclusivamente un mezzo per regolare gli scambi, "e dedicarsi allo studio di un sistema in cui la moneta influenza l'andamento complessivo, compresi i livelli della produzione e dell'occupazione" 34 . Tuttavia, se il livello della produzione non è un dato indipendente dalla situazione monetaria, viene meno il presupposto della piena occupazione delle risorse. Cade quindi anche la teoria quantitativa della moneta. Il passo successivo è allora "l'indagine su che cosa realmente determini, in ciascun momento, il livello effettivo dell'attività produttiva... Prese avvio da qui il viaggio interminabile nella teoria dell'offerta di breve periodo...e dopo una laboriosa gestazione, la General Theory" 35 .

Sono di questo periodo alcuni scritti sui limiti del capitalismo ed alcune proposte di politica economica basate su un maggiore intervento dello Stato a sostegno dell'occupazione ${ }^{36}$. L'obiettivo era dimostrare "come gli effetti positivi dei programmi di lavori pubblici venissero generalmente sottovalutati: oltre al numero di lavoratori assorbiti direttamente in tali programmi, si doveva tener conto di quelli occupati nelle industrie che producevano o fornivano i materiali necessari alla loro realizzazione. Bisognava, in altre parole, considerare anche gli effetti indiretti, ma soprattutto, aggiunse Keynes, il maggior reddito così generato... avrebbe determinato una maggior domanda di beni di consumo e quindi un ulteriore aumento dell'occupazione nelle industrie in grado di soddisfare la nuova domanda" ${ }^{37}$. Stava nascendo l'idea del moltiplicatore. Un passo importante in questa direzione venne da un

33 J.M. KEYNES, Teoria generale dell'occupazione, dell'interesse e della moneta e altri scritti, cit., p. XXXIII.

34 J.M. KEYNES, Teoria generale dell'occupazione, dell'interesse e della moneta e altri scritti, cit., p. XXXIV.

35 J.M. KEYNES, Teoria generale dell'occupazione, dell'interesse e della moneta e altri scritti, cit., p. XXXIV.

36 J.M. Keynes, H. Henderson, Can Lloyd George Do It?, An Examination of the liberal Pledge, London, in "The Nation and Athenaeum", 1929.

37 J.M. KEYNES, Teoria generale dell'occupazione, dell'interesse e della moneta e altri scritti, cit., p. XLV. 
saggio di Richard Kahn, che fu il primo a spiegarne il funzionamento e a evidenziarne la carica innovativa ${ }^{38}$. Keynes propose di chiamare questo meccanismo il "moltiplicatore degli investimenti". "È questo "uno dei cardini della nuova teoria della determinazione del reddito che avrebbe trovato la formulazione definitiva nella General Theory.... un'iniziale spesa di investimento avrebbe prodotto sicuramente un volume di reddito e un livello di occupazione maggiori del suo effetto primario" 39 . Veniva così modificato radicalmente il modo di concepire il funzionamento del sistema economico: il centro dell'analisi dell'equilibrio non erano cioè più l'offerta e la disponibilità di fattori produttivi, ma la loro domanda. Cruciale diventava il ruolo degli investimenti. Con il moltiplicatore venivano introdotti per la prima volta nell'analisi economica anche fattori di natura psicologica. Si noti tuttavia che fu solo nel 1934 che Keynes illustrò con chiarezza il proprio attacco alla teoria classica in un intervento radiofonico alla $\mathrm{BBC}^{40}$.

All'indomani della pubblicazione della General Theory i giudizi dei maggiori economisti, coetanei o più anziani di Keynes, furono prevalentemente negativi. Frank Knight scrisse: "È spaventosamente difficile capire che cosa l'autore intenda dire [...] Apparentemente si tratta di un equilbrio con forte disoccupazione involontaria [...]. Ma io non riesco a prendere questo nuovo e rivoluzionario equilibrio sul serio... Debbo confessare che il lavoro che mi è costato la lettura di The General Theory... mi lascia con un senso di acuta delusione. Il principale valore del libro mi è sembrato consistere nella fatica di leggerlo" 41 . Anche per Schumpeter la Teoria generale non conteneva nulla di nuovo rispetto alle politiche che Keynes proponeva da anni e "altro non era se non l'espressione di una civiltà decadente" 42 . Fra tutte la recensione più dura fu quella di Pigou. Il libro si fondava secondo lui su "defini-

${ }^{38}$ R.F. KAHN, The relation of Home Investment to Unemployment, in "Economic Journal", 162, 1931, pp. 137-198.

39 J.M. KeYNES, Teoria generale dell'occupazione, dell'interesse e della moneta e altri scritti, cit., p. XLVII.

40 Il testo, dal titolo Poverty in Plenty: Is the Economic System Self-Adjusting? è incluso in questo volume, pp. 570-577.

${ }^{41}$ F.H. KNIGHT, Unemployment and Mr Keynes' Revolution in Economic Theory, in "Canadian Journal of Economics and Political Science", 1, 1937, p. 121, p. 123.

42 J. A. SChumpeter, The General Theory of Employment, Interest and Money, in "Journal of the American Statistical Association", n. 196, 1936, p. 791. 
zioni imprecise e conclusioni non sufficientemente dimostrate"43: il giudizio, tuttavia, venne rivisto e corretto nel 1949, tre anni dopo la morte di Keynes ${ }^{44}$.

In Italia l'accoglienza di Keynes fu differente a seconda che ci si collocasse prima o dopo la pubblicazione della Teoria Generale ${ }^{45}$. Negli anni Venti e Trenta del Novecento le prime opere di Keynes avevano avuto in Italia pronto e largo consenso, grazie anche alle traduzioni pubblicate dai fratelli Treves ${ }^{46}$. Einaudi aveva contribuito alla divulgazione e alla discussione delle tesi keynesiane in tema di politica monetaria in alcuni saggi apparsi nel 1933 sulla "Riforma Sociale". Ben diversa, invece, è stata l'accoglienza della Teoria generale. "L'acclimatamento del pensiero di Keynes" in Italia (per usare la felice espressione di Caffè ripresa da Becattini) fu infatti un processo lungo e laborioso ${ }^{47}$. Agiva come ostacolo all'introduzione di Keynes nel panorama teorico italiano proprio l'alta tradizione e la robustezza del pensiero economico nazionale. Era questa una questione ben chiara allo stesso Keynes: "In economia tu non puoi convincere il tuo avversario, se la sua testa è già così satura di concetti avversi che egli non riesce a cogliere le chiavi del pensiero che tu gli offri" 48 . Di Nardi nel Convegno del 1983 su Keynes in Italia fu esplicito a questo proposito: "La ragione della cauta accoglienza riservata alla Teoria generale sarebbe spiegabile, per un verso, con la familiarità della teoria dell'equilibrio economico generale fra gli economisti italiani e, per altro verso, con le riserve espresse da alcuni autorevoli Maestri italiani (Einaudi, Bresciani Tur-

43 A.C. Pigou, Mr J, M. Keyne's General Theory of Employment, Interest and Money, in "Economica", n. 10, 1936, p. 115.

${ }^{44}$ J.M. KEYNES, Teoria generale dell'occupazione, dell'interesse e della moneta e altri scritti, cit., p. LXX.

45 Per una più approfondita analisi di questo tema si rimanda a G. LunghINI, R. TARGetti Lenti, Di Fenizio e l'economia politica di Keynes, in "Il Politico", n. 2, 2003, pp. 285-306.

46 Il saggio Le conseguenze economiche della pace (1919) era stato tradotto da V. Tasco e pubblicato nel 1920. La riforma monetaria (1923) era stato tradotto da P. Sraffa e pubblicato nel 1925. I due volumi del Trattato della moneta (1930) furono tradotti da E. Radaelli e pubblicati, rispettivamente, nel 1932 e nel 1934.

${ }^{47} \mathrm{G}$. BeCATtinI, L'acclimatamento del pensiero di Keynes in Italia: invito a un dibattito, in: A.A.V.V., " Atti del Convegno Keynes in Italia”, Università degli Studi di Firenze, 4-5 giugno 1983, Annali dell'Economia Italiana, Istituto IPSOA, 1984.

48 J.M. KeYNes, The Collected Writings, vol. XIII, 1977, p. 470. 
roni, Papi, Demaria) sulla capacità esplicativa del modello keynesiano"49. Più radicale è la critica di Demaria con riferimento alla "mancata generalità del pensiero keynesiano, generalità che nelle teorie dell'equilibrio economico generale è di importanza vitale quale base di qualsiasi ragionamento economico. Questa incapacità di guardare più lontano pregiudica non solo la trattazione dei problemi monetari di indole teorica, ma anche quella dei problemi di politica economica" 50 .

Alle resistenze nei confronti del pensiero keynesiano contribuì non poco la diffidenza manifestata da Einaudi nei confronti di alcuni degli schemi interpretativi keynesiani, e in particolare della relazione tra risparmi e investimenti. Einaudi era stato d'altra parte influenzato da $\mathrm{Pa}-$ reto e da Pantaleoni e fondava i propri riferimenti teorici nella teoria dell'equilibrio economico generale. Tra i contributi più significativi di critica dell'impostazione keynesiana merita di essere ricordato, in particolare, il saggio in cui viene ricostruita la polemica intercorsa nel 1933 tra Einaudi e Pagni in seguito alla ricostruzione del pensiero di Keynes fatta da Pagni su "La Riforma sociale" "per mostrarne l'efficacia operativa in quel particolare momento di crisi economica". Einaudi era convinto che non si potesse creare occupazione durevole grazie a una spesa pubblica in disavanzo e senza dover pagare un prezzo in termini di inflazione, di riduzione degli investimenti produttivi e di rallentamento della crescita economica. Era anzi convinto che senza lepri non si possano fare pasticci di lepre, che cioè senza vero risparmio non si possano fare investimenti51. Einaudi condivideva con altri grandi economisti del suo tempo la convinzione che il comportamento virtuoso da promuovere per il buon funzionamento del sistema economico sia incentivare il risparmio sia privato che pubblico. La parsimonia degli individui era considerata una virtù così come il pareggio o addirittura il surplus del bilancio pubblico era considerato un segnale della solidità di uno Stato;

${ }^{49}$ G. Di NARDI, Keynes in Italia: perché incontrò resistenza, in A.A.V.V., " Atti del Convegno Keynes in Italia", Università degli Studi di Firenze, 4-5 giugno 1983, Annali dell'Economia Italiana, Istituto IPSOA, 1984, p. 46.

50 G. Demaria, Testimonianza, in A.A.v.v., " Atti del Convegno Keynes in Italia", Università degli Studi di Firenze, 4-5 giugno 1983, Annali dell'Economia Italiana, Istituto IPSOA, 1984, p. 41.

${ }^{51}$ L. EINAUDI, Il mio piano non è quello di Keynes, in "La Riforma sociale", marzoaprie, 1933, pp. 129-142; L. EINAUdI, C. PAGNI, Fondo disponibile di risparmio e lavori pubblici, in "La Riforma sociale", maggio-giugno, 1933, pp. 331-352. 
mentre la spesa in disavanzo e il debito pubblico erano ritenuti un segnale di squilibrio. Keynes ribalta completamente questa visione basandosi su due considerazioni: 1) ciò che è vero per un singolo individuo può non esserlo per l'aggregato; 2) l'inversione del nesso di causalità nella catena investimento-reddito-risparmio. È la spesa autonoma che genera il reddito da cui proviene il risparmio e non viceversa. Allo stesso modo, la riduzione del debito pubblico e del disavanzo sono il risultato della crescita del reddito trainata dalla domanda effettiva, e non di una riduzione della stessa a favore del risparmio.

In verità, come nota Becattini, la diffidenza nei confronti di Keynes non riguardava tanto le sue categorie analitiche quanto piuttosto era la vena più squisitamente ideologica del suo pensiero a sollevare riserve aperte. Questa diffidenza spiega anche la ragione delle difficoltà incontrate nel trovare chi fosse disposto a tradurre la Teoria generale. Come ricorda Maria Cristina Marcuzzo, in una lettera del 1938 a Celestino Arena, Direttore della "Nuova Collana degli Economisti" della UTET, nel negoziare per incarico dello stesso Keynes i termini contrattuali della traduzione italiana della Teoria generale e di altri scritti di Keynes, Sraffa scriveva che la traduzione "sarà impresa tutt'altro che leggera" 52 . Per questo rifiutò e alla fine la traduzione venne affidata ad Alberto Campolongo e pubblicata nel 194753. Tradurre Keynes, osserva La Malfa, non presenta difficoltà di lingua. È difficile, invece, capire la natura delle sue argomentazioni e la ragione di concetti del tutto nuovi e non convenzionali. Sempre La Malfa riporta il pensiero di Virginia Woolf su Keynes: “...come gli ho detto quando mi ha dato da leggere alcune pagine del suo nuovo libro, i processi mentali che vi sono contenuti sono molto più avanti dei miei, come lo sono quelli di Shakespeare" 54 .

Durante i primi trent'anni del dopoguerra il modello interpretativo keynesiano è stato predominante nella spiegazione del funzionamento delle economie capitalistiche e nella formulazione delle politiche eco-

52 M.C. Marcuzzo, Keynes, perché la sua saggezza è sempre attuale, presentazione del volume di Giorgio La Malfa, Roma, Accademia dei Lincei, 23 maggio 2019.

53 J.M. KeYNES, Occupazione, interesse e moneta: teoria generale, traduzione di A. Campolongo, Torino, UTET, 1947.

54 J.M. KEYNES, Teoria generale dell'occupazione, dell'interesse e della moneta e altri scritti, cit., p. CLIII. 
nomiche. A partire dall'inizio degli anni Ottanta del secolo scorso, tuttavia, le interpretazioni di contenuto keynesiano sono state sostituite dai modelli di stampo neoclassico. Solamente con la crisi finanziaria del 2007 - 2008 e con il crollo dei mercati subprime negli Stati Uniti, si è reso necessario un ripensamento del funzionamento dei sistemi economici di mercato. L'analisi di stampo keynesiano ha ripreso un certo vigore nella consapevolezza che interventi correttivi dello Stato siano necessari quando il mercato da solo non riesce ad assicurare livelli di piena occupazione. La "rivoluzione keynesiana" potrebbe quindi fornire ancora oggi risposte adeguate ai problemi delle società contemporanee, dove sempre più è necessario combinare efficienza ed equità. In base a questi modelli interpretativi la depressione dovrebbe essere combattuta con politiche di sostegno della domanda e non, invece, con politiche di austerità o seguendo i precetti della supply side economics suggeriti dai liberisti.

Diceva già a suo tempo Keynes: "Se il nuovo problema [la tendenza alla depressione economica] non verrà risolto l'ordine attuale della società sarà così screditato che diventeranno inevitabili cambiamenti selvaggi, sconsiderati e distruttivi" 55 . I cambiamenti tuttavia, vanno attentamente considerati, per valutarne le conseguenze, e vanno introdotti con gradualità. "I difetti economici pù evidenti della società in cui viviamo sono l'incapacità di assicurare la piena occupazione e la sua arbitraria e iniqua distribuzione della ricchezza e dei redditi.... la crescita della ricchezza, lungi dal dipendere....dalla parsimonia dei ricchi, al contrario ne è danneggiata... Lo Stato dovrà assumere un ruolo di guida nell'influenzare la propensione al consumo...Penso quindi che una socializzazione piuttosto estesa degli investimenti si dimostrerà il solo mezzo per realizzare una situazione che si approssimi alla piena occupazione......La critica che abbiamo mosso alla teoria economica classica...è consistita non tanto nell'individuarvi errori logici, quanto nel porre in rilievo che essa si basa tacitamente su presupposti che non sono mai o quasi mai soddisfatti, e che di conseguenza non è in grado di risolvere i problemi economici del mondo reale" 56 .

55 J.M. KeynES, Teoria generale dell'occupazione, dell'interesse e della moneta e altri scritti, cit., p. CIV.

56 J.M. KEYNES, Teoria generale dell'occupazione, dell'interesse e della moneta e altri scritti, cit., p. 433. 
Keynes mira dunque a scongiurare nuove crisi attraverso un ruolo attivo dello Stato. Scriveva in un breve articolo dal titolo L'arte e lo Stato, anch'esso del 1936: "Perché non si dovrebbe rendere tutta Londra simile alla zona di St. James Park e ai suoi dintorni? I lungofiume potrebbero diventare uno dei più bei panorami al mondo, con una teoria di edifici e terrazze che sorgono lungo le rive....Tutti i nostri architetti, ingegneri e artisti dovrebbero avere l'opportunità di dar corpo alle diverse fantasie non già di esseri esacerbati, rachitici e disillusi, ma di spiriti sereni e soddisfatti che appartengono a un rinascimento. Affermo che non vi possono essere ostacoli di natura 'finanziaria' che impediscano queste realizzazioni, qualora siano disponibili la forza lavoro e le risorse materiali necessarie" 57 .

La Teoria generale, osserva La Malfa, non è una teoria generale dello sviluppo capitalistico: è una critica del funzionamento dei sistemi capitalistici, uno strumento utile a definire i possibili interventi correttivi di alcune patologie del sistema. In particolare, come conclude Keynes nel capitolo 24 della Teoria generale, "I difetti economici più evidenti della società in cui viviamo sono l'incapacità di assicurare la piena occupazione e la sua arbitraria e iniqua distribuzione della ricchezza e dei redditi. La relazione della teoria qui sviluppata con il primo di questi problemi è ovvia. Ma sotto due aspetti importanti è attinente anche al secondo" 58 .

Il fascino e l'attualità della visione di Keynes nasce dalla ricchezza di un pensiero capace di collocare l'economia in un ambito molto più ricco di quanto tradizionalmente avvenga, tenendo conto delle interrelazioni con la storia, la politica, la società, la filosofia. Keynes ha vissuto in un'epoca di crisi profonda segnata da ben due conflitti mondiali e dal crollo di Wall Street nel 1929. La forza del suo messaggio sta soprattutto nell'evidenziare come sia necessario ricercare e proporre politiche innovative adeguate ai mutamenti della realtà, nel rifiutare di rassegnarsi a spiegazioni superate e nel riaffermare l'idea secondo cui la scienza economica non deve tanto mirare al bene dei mercati ma a quello della collettività, recuperando quell'umanesimo che tanto gli

57 J.M. KEYNES, Teoria generale dell'occupazione, dell'interesse e della moneta e altri scritti, cit., p. 589.

58 J.M. KEYNES, Teoria generale dell'occupazione, dell'interesse e della moneta e altri scritti, cit., p. 426 . 
stava a cuore. In sintesi le argomentazioni critiche di Keynes si fondano su due punti: uno è la confutazione dell'individualismo metodologico - che suppone che ciò che è vero per un singolo individuo sia vero per l'insieme di individui (la cosiddetta fallacy of composition) - e l'altro è l'inversione del nesso di causalità nella catena investimento-redditorisparmio: è la spesa autonoma che genera il reddito da cui proviene il risparmio, per cui una generalizzata riduzione della spesa, riducendo il reddito, può addirittura rendere impossibile l'aumento desiderato del risparmio, a riprova dell'errore di ignorare l' "effetto composizione". Allo stesso modo, l'abbattimento del debito pubblico e la riduzione del disavanzo si ottengono solo con una crescita del reddito trainata dalla domanda, non con programmi di austerità, che comprimono il reddito e quindi anche il futuro gettito fiscale.

In conclusione, Keynes ci lascia una guida su come riuscire a organizzare una società migliore, stabilendo regole e mettendo limiti all'azione del mercato, impedendo che l'interesse individuale prevalga, prevedendo l' intervento pubblico quando si presenti una carenza di domanda che deprime reddito e occupazione. Riproporre la Teoria generale in una nuova traduzione, insieme a molti altri scritti di cui alcuni inediti, con il rilevante apparato critico che un'edizione dei "Meridiani" comporta, equivale a evidenziare, in un'Europa "succube" dell'austerità, la necessità di una organica, adeguata politica economica alternativa improntata alla crescita, in grado di recuperare il messaggio keynesiano. Giorgio La Malfa è convinto che si debba "tornare" a Keynes non soltanto perché le sue idee economiche sono state nascoste per troppi anni dalla ripresa delle concezioni tradizionali che affidano ai mercati la soluzione dei problemi e guardano con diffidenza all'azione collettiva, ma soprattutto per l'atteggiamento di apertura di questo autore rispetto ai problemi sociali. "Keynes ha fatto la sua parte nel riempire l'agenda di contenuti validi per il suo tempo. Toccherebbe ora all'economia e alla politica contemporanee individuare la risposta ai problemi del nostro tempo e delineare la saggezza nuova per una nuova era" 59 .

59 J.M. KEYNES, Teoria generale dell'occupazione, dell'interesse e della moneta e altri scritti, cit., p. CXII. 


\begin{abstract}
The "General Theory of Employment, Interest and Money" is presented here through the translation by Giorgio La Malfa, with an introduction which highlights not only its historical and theoretical backgrounds, but also its links with the greatest thinkers of the time, from Freud, Wittgenstein and Einstein to Virginia Woolf, Thomas Eliot and the other members of the Bloomsbury circle. The volume also collects a group of writings published before and after the "General Theory", closely related to it, including the author's preface to the various foreign editions.Keynes was the thinker of
\end{abstract}

an era of deep crisis. The Great War, the collapse of Wall Street in 1929, the Second World War dramatically marked its time, and the strength of his message not only consists in offering an explanation of the historical and political causes of that dramatic season, but also in assessing a new "vision" of the economy highly connected with history, politics and society. Giorgio La Malfa claims that today, above all in Italy and in Europe, it is necessary to recover the keynesian message and to adopt keynesian economic policies aimed at stimulating growth and employment. 